\title{
Concepciones sobre calidad de vida laboral en las organizaciones ${ }^{13}$
}

\author{
Martín Eduardo Salas Ibarra \\ Psicólogo \\ Universidad de Nariño, Colombia \\ Correo electrónico: salas5840@gmail.com \\ Yesica Meliza Basante Pantoja \\ Psicóloga \\ Universidad de Nariño, Colombia \\ Correo electrónico: melibasante19@gmail.com
}

\section{Christian Alexander Zambrano Guerrero \\ PhD. en Ciencias de la Educación (c) \\ Universidad de Nariño, Colombia \\ Correo electrónico: cazambranopsi@gmail.com}
Sonia Maritza Matabanchoy Tulcan
PhD. Psicología
Universidad de Nariño, Colombia
Correo electrónico: somapsicologa@yahoo.es

\section{Armando David Narváez Chaves \\ Psicólogo \\ Universidad de Nariño, Colombia \\ Correo electrónico: dave.narvaez97@gmail.com}

Recibido: 22/11/2019

Evaluado: $10 / 02 / 2020$

Aceptado: 01/04/2020

\section{Resumen}

El presente artículo tiene por objetivo analizar las concepciones sobre Calidad de Vida Laboral (CVL) en las organizaciones, a través de la revisión de distintas investigaciones encontradas en bases de datos especializadas durante los últimos 10 años. El estudio es una revisión sistemática (RS) descriptiva de literatura, siguiendo las directrices de la declaración PRISMA. En total se revisaron y analizaron 63 artículos de las bases de datos ScienceDirect, SciELO, Dialnet y Redalyc, que abordan las concepciones acerca de CVL en las organizaciones. Como conclusión, se menciona que la CVL se considera una categoría de análisis relativamente joven, que presenta variedad de definiciones operativas, que resultan por sí solas insuficientes para articular modelos teóricos bajo una única perspectiva; así, es necesario continuar con la investigación sobre CVL, para ampliar y fundamentar a través del estudio empírico una comprensión más clara, dado que esta categoría puede propender a la humanización de los puestos de trabajo y a mejorar la dignidad de los colaboradores.

Palabras clave Calidad de vida laboral, colaboradores, organizaciones, trabajo.

13 Para citar este artículo: Salas, M., Basante, Y., Zambrano, C., Matabanchoy, S. y Narváez, A. (2021). Concepciones sobre Calidad de Vida Laboral en las organizaciones. Informes Psicológicos, 21(2), pp. 209-227 http://dx.doi.org/10.18566/infpsic.v21n2a13 


\title{
Conceptions about Quality of Work Life in organizations
}

\begin{abstract}
The objective of this article is to analyze the conceptions on Quality of Work Life (QWL) in organizations through the review of different studies found in specialized databases during the last 10 years. The study is a descriptive systematic review (SR) of literature, following the guidelines of the PRISMA statement. In total, 63 articles from the ScienceDirect, SciELO, Dialnet and Redalyc databases were reviewed and analyzed, addressing the conceptions about QWL in organizations. It can be concluded that the QWL is considered a relatively new category of analysis that presents a variety of operational definitions, which are by themselves insufficient to articulate theoretical models under a single perspective. Thus, it is paramount to continue doing research on QWL, to expand and support a clearer understanding of the topic through empirical study, since this category can lead to the humanization of jobs and to improve the dignity of employees.
\end{abstract}

Keywords Quality of work life, collaborators, organizations, work.

\section{Concepções sobre Qualidade de Vida no Trabalho nas organizações}

\section{Resumo}

0 objetivo deste artigo é analisar as concepções sobre Qualidade de Vida no Trabalho (CVL) nas organizações, por meio da revisão de diferentes investigações encontradas em bases de dados especializadas nos últimos 10 anos. 0 estudo é uma revisão sistemática descritiva (RS) da literatura, seguindo as diretrizes do PRISMA. No total, foram revisados e analisados 63 artigos das bases de dados ScienceDirect, SciELO, Dialnet e Redalyc, abordando as concepções sobre a CVL nas organizações. Como conclusão, menciona-se que a CVL é considerada uma categoria de análise relativamente jovem, que apresenta uma variedade de definições operacionais, que por si só são insuficientes para articular modelos teóricos sob uma única perspectiva; Assim, é necessário dar continuidade às pesquisas sobre a CVL, para ampliar e subsidiar um entendimento mais claro por meio do estudo empírico, uma vez que esta categoria pode levar à humanização do trabalho e à dignidade dos funcionários.

\section{Palavras chave}

Qualidade de vida no trabalho, colaboradores, organizações, trabalho. 


\section{ntroducción}

El concepto de Calidad de Vida Laboral (CVL) nace de una serie de conferencias desarrolladas por el Ministerio de Trabajo de los Estados Unidos en la década de los setenta y se relaciona con la satisfacción que los colaboradores experimentan en su trabajo (Granados, 2011). Por otra parte, se tiene en cuenta el incremento de la autonomía, esto con el propósito de fomentar procesos de aprendizaje y humanización, incrementando la participación laboral (Walton, 1973). En los años ochenta la CVL enmarca nuevas dinámicas, a través de procesos como la eficacia organizacional, la participación y la toma de decisiones (Nadler \& Lawler, 1983). En los años noventa la CVL involucra la gestión del recurso humano, fortaleciendo el compromiso, los conocimientos y habilidades de los trabajadores; además de reconocer los derechos laborales y la protección social, con la ayuda de las acciones elaboradas desde la seguridad y salud en el trabajo (SST) (Blanch, 2011).

Desde el año 2000 al 2009, la CVL aumenta su relación con la satisfacción y el bienestar, reforzando la comunicación entre compañeros de trabajo; esto conlleva al crecimiento de la productividad, posicionamiento y competitividad de las distintas organizaciones en el mercado empresarial (Segurado \& Agulló, 2002). Según Rodríguez (2012), a partir del año 2010 se evidencia un incremento en la responsabilidad social empresarial desde la SST. Así mismo se consolidan los requerimientos de protección y acompañamiento al recurso humano (Organización Internacional del Trabajo [OIT], 2019).

De esta forma, hoy en día es necesario considerar la CVL como un componente necesario para el desarrollo integral de los colaboradores desde las distintas políticas organizacionales; de esta manera, se retoma la teoría de demandas y recursos laborales que, a su vez, considera elementos que permiten la prevención del burnout, aumentando el compromiso y la conexión con el trabajo, siendo esta teoría predictora y explicativa de las distintas variables relacionadas con la CVL (Bakker \& Demerouti, 2013).

La CVL, según Caballero y Nieto (2015), se tiende a asociar con distintas variables organizacionales como: a) el bienestar laboral $(B L)$ entendido, según Echevarría y Santiago (2017), como la unión de factores tanto biológicos psicológicos y sociales de los trabajadores, frente a las dinámicas de los contextos laborales, con respuestas emocionales y conductuales positivas; b) la satisfacción laboral (SL), definida como una muestra de sentimientos positivos de los trabajadores respecto a las responsabilidades laborales con una adecuada adaptación a las dinámicas organizacionales (Montoya, Méndez \& Boyero, 2017); c) el engagement (ENG) comprendido como un mayor grado de involucramiento y compromiso de los colaboradores frente a sus funciones dentro de las empresas, desempeñando sus actividades con más vigor y energía, con sentimientos de orgullo e inspiración por su trabajo (Marcantonio, 2017); y d) la dignificación y humanización del trabajo (DHT), que busca 
guiar el respeto por los valores de las personas dentro de los contextos laborales, siempre en concordancia con la ética y tolerancia por las costumbres y la dignidad, siendo ésta inviolable (Carlosama et al., 2019).

Dada la relación con distintas variables, se dificulta la definición y operacionalización de la CVL, debido a la pluralidad de dimensiones transversales al límite organizacional y laboral, en donde existe variedad de disciplinas, enfoques teóricos y áreas de estudio. Por lo tanto, al no existir un concepto claro y unificado, los programas de evaluación, intervención y seguimiento acerca de esta temática, pueden no responder a un objetivo concreto (Granados, 2011).

De esta manera, se identifica la necesidad de realizar una RS sobre el concepto de CVL, dado que una definición precisa se configura en un reto. Por otro lado, una RS se constituye como herramienta esencial para sintetizar la información científica disponible acerca de un tema específico (Ferreira, Urrutia \& Coello, 2011), por ende, esta temática es relevante dada la inquietud de las organizaciones por evaluar los sentimientos y actitudes de los colaboradores referente a las actividades laborales. Para ello, es necesario fomentar la creación de programas de intervención y seguimiento que favorezcan la disminución del impacto de los riesgos psicosociales, facilitando respuestas cognitivas, emocionales y conductuales adecuadas ante unas condiciones de trabajo cada día más exigentes (Palomo, Carrasco, Bastias, Méndez \& Jiménez, 2015). Igualmente, Carlosama et al. (2019) precisan fortalecer el respeto por la dignidad, ampliando el interés por DHT, guiados siempre por los principios éticos y morales de cada intervención. De esta manera, es pertinente comprender la CVL desde distintos conceptos organizacionales con los cuales se relaciona, vislumbrando el aporte académico que ha tenido esta temática.

Por consiguiente, el objetivo de este trabajo está dirigido al análisis de la CVL en las organizaciones, a través de la revisión de distintas investigaciones encontradas en bases de datos especializadas durante los últimos 10 años y sintetizadas mediante las directrices de la declaración PRISMA, utilizada para mejorar la exhaustividad de los distintos trabajos de RS, desde su planeación hasta el desarrollo de los resultados encontrados, por medio de los criterios de elegibilidad convenientes para cada investigación (Hutton, Catalá \& Moher, 2016).

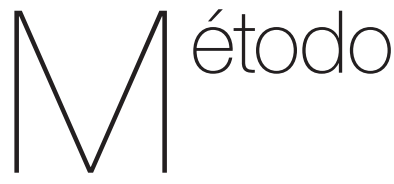

El presente estudio es de tipo descriptivo a partir de estudios originales empíricos (Ferreira et al., 2011). Se realizó una RS descriptiva de literatura, observacional y retrospectiva. Se analizaron 63 artículos por contar en su introducción, metodología, resultados, discusión y conclusiones, variadas concepciones acerca de CVL en las organizaciones. 
Se siguieron las directrices de la declaración PRISMA recomendada para el adelanto de revisiones bibliográficas sistemáticas que incorporan metaanálisis en red (Hutton et al., 2016), las cuales aseguraron que los artículos incluidos fueran revisados en su totalidad de forma clara y precisa. Para este propósito se usaron 6 ítems de la lista de control a partir de su pertinencia y detalle de los requisitos de selección para cada sección:1) Introducción; Razón fundamental; (2) Objetivos, Sección Métodos; (3) Criterios de elegibilidad; (4) Fuentes de información; (5) Estrategia de búsqueda; y (6) Elementos de datos, criterios de inclusión y exclusión de cada artículo. Para la recolección de datos se usó una hoja electrónica creada para el estudio, donde los datos considerados fueron: año de publicación, país, idioma, objetivo, metodología, concepciones de CVL y resultados.
Los estudios se recuperaron de las bases de datos ScienceDirect, uno de los índices especializados a nivel mundial de mayor calidad; SciELO, que ofrece índices y publicaciones periódicas académicas que cubren diferentes áreas de las ciencias; Dialnet, que brinda mayor visibilidad en los ámbitos de las ciencias humanas, jurídicas administrativas y sociales; $y$, finalmente, Redalyc, que conserva una naturaleza académica extensa. Es así como se concluyó que estas bases proporcionan un estándar académico de alta calidad y oportuno para la búsqueda de artículos relacionados con CVL, además de proporcionar acceso directo a la gran mayoría de estudios. La búsqueda y análisis se realizó entre los meses de febrero y agosto de 2019.

El procedimiento de la presente investigación se expone a continuación, a través del desarrollo de diez etapas.

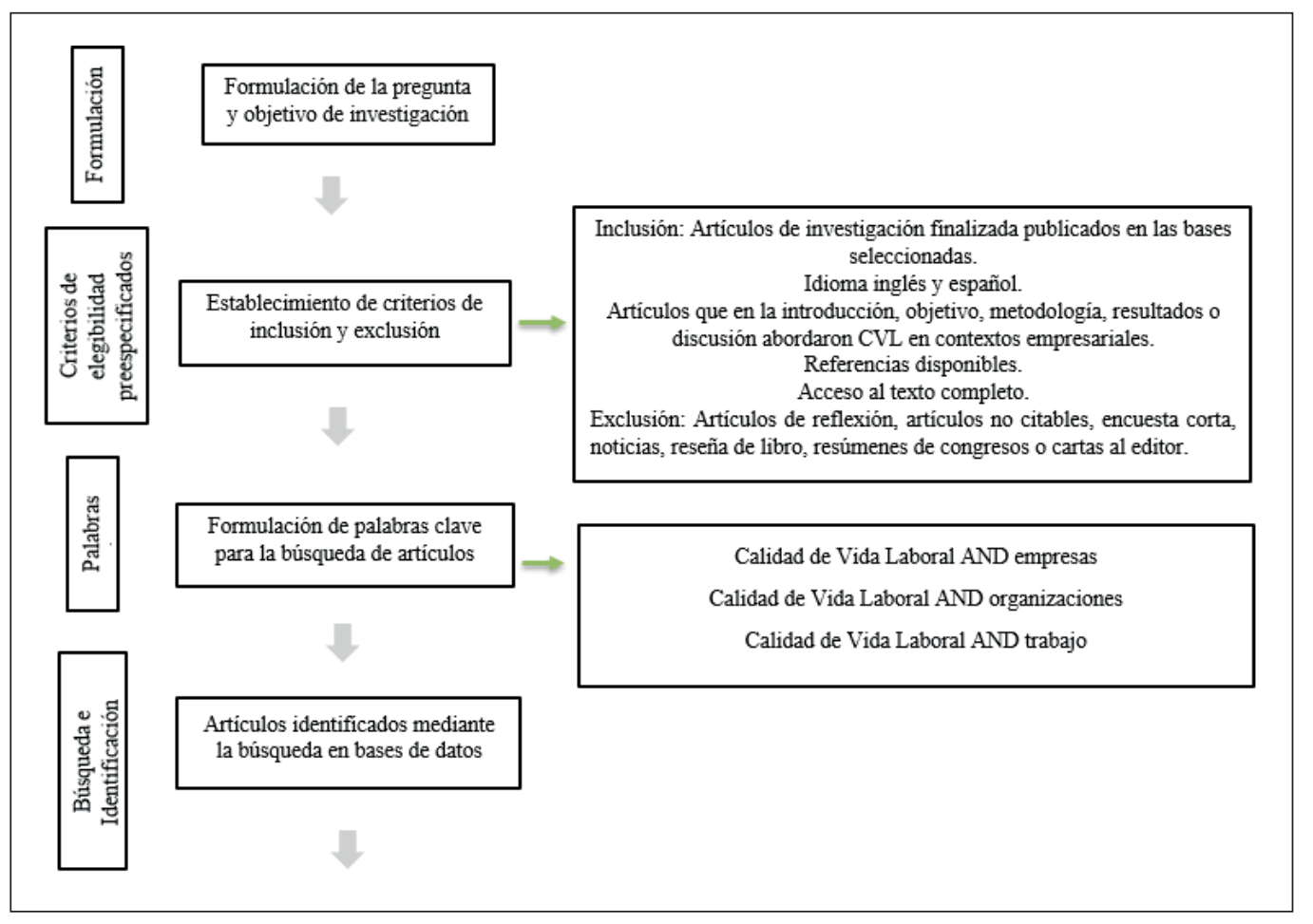


Número de artículos seleccionados para evaluación con criterios de matriz Prisma

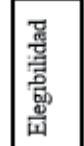

营

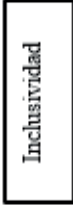

$\frac{n}{y}$

Interpretación y análisis de

resultados
Número de artículos incluidos:

89

1)Título, 2) Año de publicación, 3) Pais, 4) Idioma, 5) Objetivo, 6) Concepción de CVL, 7) Resultados. Evaluación por elegibilidad de
acuerdo a aplicación de criterios $\Rightarrow \quad \begin{gathered}\text { 1)introduccion, Razon fundamental, 2) Objetivos, 3) } \\ \text { Criterios de elegibilidad, 4) Fuentes de información, 5) }\end{gathered}$ Estrategia de búsqueda y 6) Elementos de datos

Número de artículos incluidos:

63

Número de artículos excluidos:

Figura 1. Procedimiento por etapas.

Nota: Elaboración propia.

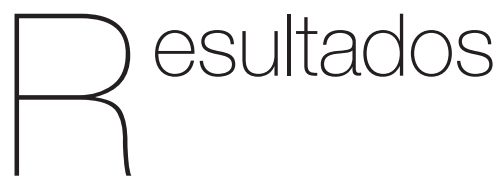

Se presentan a continuación los resultados, teniendo en cuenta idioma, año y país de publicación, además de la metodología utilizada en cada uno de los artículos revisados, así como los sectores donde se ha trabajado la CVL. Finalmente se exponen las concepciones acerca de CVL agrupadas en categorías.

\section{Año de publicación, país e idioma.}

Se encontró que el 2017 fue el año con más publicaciones relacionadas con CVL con un 20.6\%; $(n=13)$ y los años 2012 y 2019 fueron los años que presentaron menos publicaciones con un 1.5\%; $(n=1)$ cada uno. Además, se identificaron 11 países donde se ha trabajado esta temática, siendo Colombia el país con mayor publicaciones con un 34.9\%; $(n=22)$, seguido de España, con un 22.2\% ( $n=14)$, mientras que Argentina, Guatemala, Uruguay, Brasil 
y Ecuador evidenciaron el menor número de publicaciones, con un $1.5 \%(n=1)$ cada uno. En cuanto a las publicaciones en idioma español se destaca un $71.4 \%(n=45)$ y en inglés un $28.6 \%(n=18)$.

\section{Metodologías utilizadas}

Respecto a las metodología utilizadas, el $85.7 \%(n=54)$ de artículos pertenecen al paradigma cuantitativo, de los cuales la mayoría son de tipo descriptivo (27.7\%; n $=15)$, seguido del correlacional (14.8\%; $n$ = 8); así mismo, el 6.3\% $(n=4)$ presenta un paradigma cualitativo, trabajado desde los enfoques biográfico narrativo (3.1\%; $n$ $=2)$, hermenéutico $(1.5 \%, n=1)$, fenomenológico (1.5\%; $n=1)$; $y$, finalmente, un 7.9\% ( $n=5)$ pertenece al paradigma mixto con diseños concurrente, multifacético $(4.7 \% ; n=3)$ y secuencial $(1.5 \% ; n=1)$. Se destaca que la mayoría de los artículos con paradigma cuantitativo (31.7\%; n = 20) miden la CVL con el Cuestionario de Calidad de Vida Profesional (CVP- 351) y un $12.6 \%$ ( $n=8)$ con el Cuestionario CVT-GOHISALO; mientras que las técnicas cualitativas más utilizadas son la entrevista semiestructurada $(7.9 \% ; n=5)$ y el grupo focal $(6.3 \% ; n=4)$.

\section{Sectores donde se ha trabajado la CVL}

En cuanto a los sectores donde se ha abordado la CVL se encontró que el $41.2 \%$ ( $n=26)$ se ha trabajado en el sector salud, mayormente en personal de enfermería; un $14.2 \%(n=9)$ en el sector educativo, con mayor prevalencia en la población de docentes. De igual manera, el $14.2 \%(n=9)$ en empresas de diversos sectores productivos: un 6.3\% $(n=4)$ en el sector transporte urbano (conductores, operadores, controles de vía), un 6.3\% (n $=4$ ) en trabajadores con discapacidad intelectual y un 1.5\% ( $n=1)$ en sectores administrativos, empresas de telecomunicaciones, de vigilancia, de turismo, personal aeronáutico y profesionales de maestría y doctorado.

Se distinguieron 63 concepciones de CVL, las cuales, de acuerdo al análisis y síntesis de los autores, se lograron consolidar en 27 definiciones que se presentan en la primera columna de las Tablas 1, 2, 3 y 4 de las siguientes categorías: BL, SL, ENG y DHT. Además, en la segunda columna se encuentran las frecuencias (Fi) de cada definición y, finalmente, en la tercera columna se expone el porcentaje (\%) al que corresponde cada Fi.

Tabla 1.

Categoría Bienestar Laboral (BL)

\begin{tabular}{lcc}
\hline \multicolumn{1}{c}{ Concepciones acerca de CVL } & Fi & $\%$ \\
\hline $\begin{array}{l}\text { Grado de bienestar físico, social, económico, psicológico y cultural que experimentan las personas en su } \\
\text { entorno de trabajo, abarcando dos dimensiones, objetiva y subjetiva (Figueroa, Bastidas, Gonzales, Zam- } \\
\text { brano \& Matabanchoy, 2017; Montoya et al., 2017; Salazar, 2018). }\end{array}$ & 7 & 11.1 \\
\hline
\end{tabular}

6 El cuestionario CVP-35 realiza una medida multidimensional de la CVP. Consta de 35 preguntas que se responden en una escala de 1 a 10, y da una medida resumen de la percepción de la CVP.

EI CVT- GOHISALO, destinado a evaluar la calidad de vida en ambientes de trabajo, consta de 74 ítems y 7 dimensiones: soporte institucional, seguridad en el trabajo, integración al puesto de trabajo, satisfacción por el trabajo, bienestar logrado a través del trabajo, desarrollo personal, y administración del tiempo libre. 
Experiencia de bienestar derivada del equilibrio que percibe el colaborador, entre las demandas del trabajo y los recursos psicológicos, organizacionales y relacionales que dispone para afrontarlas (Cruz, 2018; Fernández, Cuairán \& Curbelo, 2016; Pérez, Campos, Negro \& Caballero, 2011; Rivera, Rivera \& Gonzales, 2017).

Proceso dinámico y continuo, que se interesa por el potencial y promueve el desarrollo integral de los colaboradores, en el marco de las políticas empresariales y el respeto por los derechos humanos básicos (Cruz, 2018; Hernández et al., 2017; Montoya et al., 2017).

Involucra aspectos como: compensación justa, condiciones de seguridad y salud en el trabajo, oportunidades para el continuo desarrollo laboral, integración social en la organización, normas y reglamentos de trabajo y equilibrio entre la vida laboral y familiar (Gómez, 2010; Gómez \& Ponce, 2010; Platán, 2016).

Esfuerzo por mejorar el bienestar de los empleados y la protección en su entorno de trabajo, a fin de incrementar la productividad (Hernández et al., 2017; Valero \& Riaño, 2017).

Preocupación por el bienestar y la salud de los trabajadores para el desempeño óptimo de tareas, logrando equilibrio laboral, social y familiar (García, \& Forero 2016; Gómez, 2010; Pérez, Peralta \& Fernández, 2014).

Estilos de gestión, que encaminan esfuerzos hacia el bienestar de los colaboradores y su entorno, brindando mayor autonomía, participación, toma de decisiones, responsabilidad y garantías laborales (Chaves, Grijalba, Bárcenas, Matabanchoy \& Zambrano, 2017; Martínez, Oviedo \& Luna, 2013).

Participación activa en la toma de decisiones empresariales, motivación, innovación en los sistemas de recompensa, salud y seguridad en el trabajo, otorgando oportunidades de aprendizaje para el mejoramiento del bienestar físico y psicológico (García, Gonzales \& Aldrete, 2014; Miño, 2016; Molina, Pérez, Lizárraga \& Larrañaga, 2018).

Condiciones de trabajo aceptables como la estabilidad laboral, remuneración, seguridad social y recreación, y oportunidades de desarrollo mediante el plan carrera que permiten un aumento en el bienestar del trabajador (Castro y Serna, 2016; Patlán, 2016).

Concepto multidimensional y empírico relacionado con el bienestar total, tanto físico, mental y social, en relación al contexto de trabajo y no sólo en la ausencia de enfermedad de origen laboral (Patlán, 2016; Suescún, Sarmiento, Álvarez \& Lugo, 2016).

Mantener un ambiente laboral propicio y satisfactorio para el desarrollo y bienestar, mejorando la motivación y rendimiento, potencializando las cualidades humanas de los colaboradores junto a su grupo familiar (Suescún et al., 2016; Terán \& Botero, 2011).

Concepto multidimensional que crea una percepción de necesidades cubiertas como: el soporte institucional, seguridad e integración al puesto de trabajo, identificando el bienestar a través de la actividad labora y el desarrollo personal (González et al., 2015).

En esta categoría se concibe a la CVL como la experiencia de bienestar físico, social, económico, psicológico y cultural que experimentan las personas en su entorno de trabajo, resultado del equilibrio que percibe el colaborador entre las demandas de trabajo y los recursos que dispone para afrontarlas; de esta manera, incluye tanto condiciones objetivas (seguridad y salud en el trabajo, compensación justa, reconocimiento a los colaboradores, oportunidades de aprendizaje y crecimiento laboral, etc.) como condiciones subjetivas (la forma como vive el trabajador, entre otras). 
Tabla 2

Categoría Satisfacción Laboral (SL)

\begin{tabular}{|c|c|c|}
\hline Concepciones acerca de CVL & $\mathrm{Fi}$ & $\%$ \\
\hline $\begin{array}{l}\text { Satisfacción que el colaborador experimenta en su trabajo y el ambiente en el que se desempeña (Blanch, } \\
\text { 2014; Chaves et al., 2017; Vidal, Oliver, Galiana \& Sansó, 2019). }\end{array}$ & 4 & 6.3 \\
\hline $\begin{array}{l}\text { Concepto subjetivo que pretende lograr que las experiencias laborales sean satisfactorias, influyendo en } \\
\text { el desenvolvimiento psicológico de los colaboradores en su entorno laboral y apuntando a la capacidad de } \\
\text { adaptación y creatividad (Cervantes, Blanch \& Hermoso, 2010; Flores, Jenaro, Gonzales \& García, 2010; } \\
\text { Pérez et al., 2014). }\end{array}$ & 3 & 4.7 \\
\hline $\begin{array}{l}\text { Grado de satisfacción de un individuo con su rol, relaciones, y deberes en el lugar de trabajo, basado en una } \\
\text { variedad de factores que lo afectan, incluyendo, variables de personalidad, expectativas familiares, cultura } \\
\text { del lugar de trabajo y normas sociales y/o culturales (Molina et al., 2018). }\end{array}$ & 2 & 3.1 \\
\hline $\begin{array}{l}\text { Satisfacción del trabajador con el trabajo y el ambiente en el que se desempeña, sensación de agrado con } \\
\text { las posibilidades de reconocimiento, crecimiento, participación activa que la organización le brinda (Macías, } \\
\text { Gutiérrez, Carmona \& Crespillo, 2016). }\end{array}$ & 5 & 7.9 \\
\hline $\begin{array}{l}\text { Perspectiva psicológica centrada en el individuo, que comprende la percepción que expresan los empleados } \\
\text { de una organización sobre su grado de satisfacción o insatisfacción con respecto al medio ambiente en el } \\
\text { que laboran (Cervantes et al., 2010; Gómez \& Ponce, 2010). }\end{array}$ & 4 & 6.3 \\
\hline $\begin{array}{l}\text { Valoración del colaborador respecto al conjunto de procesos y cambios en la dinámica organizacional, carac- } \\
\text { terizado por la identificación de la satisfacción que esta le genera (Segurado \& Agulló 2002). }\end{array}$ & 5 & 7.9 \\
\hline $\begin{array}{l}\text { Grado de satisfacción personal y profesional, viene dado por un determinado tipo de dirección, gestión, con- } \\
\text { diciones de trabajo, compensaciones, atracción e interés, sumado al nivel de logro y auto desarrollo, tanto } \\
\text { individual como de equipo (Salgado \& Leria, 2018). }\end{array}$ & 3 & 4.7 \\
\hline
\end{tabular}

En esta categoría se puede concluir que los elementos que retoman el concepto de $C V L$ se relacionan fundamentalmente con la satisfacción y agrado que los colaboradores sienten en el desarrollo de sus actividades laborales, su capacidad de adaptación y la creatividad que se requiere en la consecución de los objetivos empresariales.

Tabla 3.

Categoría Engagement (ENG)

\begin{tabular}{lcc}
\hline \multicolumn{1}{c}{ Concepciones acerca de CVL } & Fi & $\%$ \\
\hline $\begin{array}{l}\text { Experiencia personal de los empleados respecto a una mayor implicación en el ejercicio de sus responsabi- } \\
\text { lidades laborales (Armijo \& Portalanza, 2017; Segurado \& Agulló 2002) }\end{array}$ & 3 & 4.7 \\
$\begin{array}{l}\text { Estado de complacencia del trabajador, este busca no solo el bienestar material, sino una satisfacción no } \\
\text { monetaria para poder llegar a un nivel de ENG dentro de la organización y así evitar tener empleados con el } \\
\text { síndrome de burnout (Restrepo \& López, 2013). }\end{array}$ & 3 & 4.7 \\
$\begin{array}{l}\text { Se refiere al carácter positivo o negativo del entorno laboral, que influye en la intención del trabajador por } \\
\text { permanecer en la organización y el deseo de realizar cada vez mejor su trabajo, con mayor participación e } \\
\text { implicación diaria con la organización (Cetina, Escamilla, Centeno \& Aguilar, 2018). }\end{array}$ & 1 & 1.5 \\
\hline
\end{tabular}

En la categoría ENG se plantea que la CVL pretende lograr un máximo desempeño de los colaboradores en el cumplimiento de sus compromisos laborales, de tal manera que refuercen su fidelidad con la organización, sin que la remuneración monetaria sea su principal motivación. 
Tabla 4.

Categoría Dignificación y Humanización del trabajo DHT

\begin{tabular}{|c|c|c|}
\hline Concepciones acerca de CVL & $\mathrm{Fi}$ & $\%$ \\
\hline $\begin{array}{l}\text { La CVL es el proceso de humanizar el lugar de trabajo, a partir de la satisfacción de las necesidades de } \\
\text { los colaboradores (Chaves et al., 2017; Patlan, 2013). }\end{array}$ & 3 & 4.7 \\
\hline $\begin{array}{l}\text { Además de ser una filosofía, es un conjunto de principios según el cual las personas son el recurso más } \\
\text { importante de la organización, pues son capaces de hacer contribuciones valiosas y deben tratarse con } \\
\text { dignidad y respeto (Cruz, 2018). }\end{array}$ & 1 & 1.5 \\
\hline $\begin{array}{l}\text { Es una filosofía de gestión que mejora la dignidad del empleado, realiza cambios culturales y brinda } \\
\text { oportunidades de desarrollo y progreso personal (Molina et al., 2018). }\end{array}$ & 1 & 1.5 \\
\hline $\begin{array}{l}\text { Es una filosofía, un conjunto de creencias que engloban los esfuerzos por incrementar la productividad } \\
\text { y mejorar la motivación de las personas, enfatizando la participación de la gente, su dignificación y la } \\
\text { eliminación de aspectos disfuncionales de la jerarquía organizacional (Molina et al., 2018). }\end{array}$ & 1 & 1.5 \\
\hline
\end{tabular}

Como conclusión, en esta categoría se entiende que la CVL es una estrategia de gestión que considera el talento humano como el elemento más importante dentro las organizaciones, por ello, se interesa por propender la humanización de los puestos de trabajo y mejorar la dignidad de los colaboradores.

Finalmente, en el 7.9\% $(n=5)$ de los artículos no se identificó una definición clara acerca de CVL, más aún cuando las anteriores definiciones conceptualizan el término de Calidad de Vida (CV).

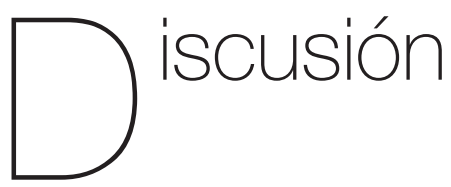

Se encontró que los países donde más estudios de la CVL se han elaborado son Colombia y España, en concordancia con un trabajo denominado CVL en Colombia: Un índice multidimensional difuso, donde el interés por el análisis de esta variable está posiblemente dirigido a que las tasas de desempleo van en disminución y el trabajo informal ha crecido sustancialmente en la última década (Gómez, Galvis \& Royuela, 2015). Por otra parte, el estudio de la CVL en España es relevante, ya que las crisis económicas que experimentan las distintas organizaciones por diversas condiciones que hacen parte de las dinámicas del mercado empresarial de los países desarrollados, suscitan un análisis más profundo de las variables que pueden incidir en estos cambios; es así como el estudio de la Universidad de Zaragoza titulado De la CVL a los riesgos Psicosociales: Evaluación de la CVL, propone una comprensión más profunda de esta variable, ya que las situaciones laborales de los trabajadores deben estar dirigidas al crecimiento y fortalecimiento de su dignidad, bienestar y satisfacción con su trabajo (Martínez \& Ros, 2010).

Respecto a la metodología utilizada en los artículos consultados se encuentra que la mayoría de las investigaciones pertenecen al paradigma cuantitativo, utilizando como instrumento para medir el Cuestionario de Calidad de Vida Profesional (CVP-35) y el Cuestionario CVT-GOHISALO, seguido de investigaciones cualitativas y mixtas, desarrollando técnicas como la entrevista semiestructurada y el grupo focal. Lo anterior 
se contradice con el estudio Tendencias de los enfoques cualitativos y cuantitativos en artículos publicados en Scientific Library On Line (SCIELO) entre los años 1997 y 2007, en donde se seleccionaron un total de 384 artículos de los cuales el $46.6 \%$ pertenecen a investigaciones cuantitativas y el $45.3 \%$ a investigaciones cualitativas, demostrando que no existe una diferencia sustancial en los trabajos según el paradigma (Cárcamo, Méndez \& Rebolledo, 2009).

Más aún, al hablar de CVL, se considera una categoría de análisis relativamente joven, que presenta variedad de definiciones operativas que resultan por sí solas insuficientes para articular modelos teóricos bajo una única perspectiva, para explicar este proceso en la vida del colaborador (Cruz, 2018; Segurado \& Agulló, 2002); así, es necesario continuar con la investigación sobre CVL ampliando y fundamentando a través del estudio empírico una comprensión más clara (Cruz, 2018).

Pese a que no existe un único método para medir la CVL, se utilizan principalmente estrategias objetivas que tratan de hacer una valoración de la misma, a través de instrumentos estandarizados que facilitan información en diferentes situaciones laborales, una aplicación rápida y masiva, así como también se logra un seguimiento y comparación de resultados para establecer diagnósticos, propuestas de intervención y evaluación de las mismas (Segurado \& Agulló, 2002).

A partir del presente estudio, se puede mencionar que la CVL se tiende a medir desde una parte objetiva (Cruz, 2018), descuidando aspectos subjetivos como las experiencias, vivencias, opiniones y juicios del colaborador; por lo tanto, desde esta perspectiva, en la psicología se están limitando los procesos de evaluación psicológica que permitan, de manera integral, utilizar herramientas cualitativas y cuantitativas hacia el estudio de la CVL, contando así con elementos insuficientes para la toma de decisiones respecto a diagnóstico, orientación, programas de promoción, intervención y seguimiento.

Respecto a los sectores donde se ha trabajado la CVL, según los artículos revisados, se encontró que la mayoría se realizaron en el sector salud, específicamente en personal de enfermería, seguido del sector educación, con mayor prevalencia en la población de docentes. Lo anterior se ratifica dado que la CVL es una variable psicosocial que implica la experiencia propia del trabajador frente al rol que desempeña, pero también a las condiciones que la organización le brinda y que son las propias de la naturaleza del cargo y de los roles que se desempeña (Pérez \& Zurita, 2016).

Para el caso del personal de enfermería, estudios como CVL en trabajadores de la salud pública en Chile describe este contexto como uno de los más complejos en relación con el contenido de las tareas, distribución del tiempo de trabajo y sobrecarga laboral (Pérez \& Zurita 2016), haciendo que las demandas cuantitativas, emocionales, ambientales y de la jornada de trabajo se configuren como factor de riesgo psicosocial. En el caso de los docentes se encontró la investigación Factores psicosociales laborales inmersos en el contexto de los docentes en una institución educativa estatal de San Juan de Pasto, donde se manifiesta que el docente se encuentra 
expuesto a demandas de carácter intralaboral (Dávila, Lombana, Matabanchoy \& Zambrano, 2018). De igual manera, el estudio CVL en hospitales y universidades mercantilizados, permite visualizar cómo en este tipo de profesionales existe deficiente control sobre el trabajo y la vida propia (Blanch, 2014), aumentado de esta forma la exposición a factores de riesgo que afectan la salud y el bienestar.

Teniendo en cuenta los resultados de la subcategoría BL, se puede inferir que los artículos revisados presentan algunas distinciones frente a la manera como abordan la CVL desde esta perspectiva; así, la mayoría de ellos lo abordan desde la mirada eudaimónica (bienestar psicológico), pues consideran que la CVL implica un proceso dinámico y continuo que se interesa por el potencial y promueve el desarrollo integral de los colaboradores. De acuerdo con Castro, Faria, Ferreira y De Fátima (2012) la perspectiva de bienestar psicológico (eudaimónico) está ligada al desarrollo del potencial humano, así como también lo corrobora Ryff (1989, citado en Romero, García \& Brustad, 2009) cuando plantea que el bienestar psicológico ha centrado su atención en el desarrollo de las capacidades y el crecimiento personal.

Asimismo, otros autores consideran que la CVL abordada desde el BL implica un equilibrio entre las demandas de la vida laboral, personal y familiar y los recursos que dispone para hacer frente a las mismas. Lo anterior se puede reafirmar puesto que el bienestar se concibe como un estado de equilibrio o balance entre de entre los desafíos (físicos, psicológicos y sociales) con los que se cuenta para enfrentarlos (Dodge et al., citado en Torres, Munguía \& Aranda,
2015). De igual manera, se encontró que algunos artículos plantean que la CVL encaminan sus esfuerzos hacia el bienestar de los colaboradores y su entorno, y ese bienestar se puede conseguir en la medida que se conceda mayores garantías laborales (Rodríguez, 2010).

A partir de la diversidad encontrada en los conceptos de la subcategoría SL, se determinaron diferentes variaciones centradas en reconocer la complacencia de los colaboradores en relación a sus actividades laborales, la manera como a partir de sus representaciones cognitivas y emocionales derivadas del reconocimiento y participación recibida permitan mayor acomodación a sus condiciones laborales, facilitando el cumplimiento de las metas planteadas con anterioridad. Se identificaron algunos elementos en común con la de definición clásica de Mobley, Griffeth, Hand y Meglino (1979), quienes equiparan la SL con las distintas respuestas afectivas que son el resultado del análisis de los trabajadores en relación a las diversas condiciones organizacionales. Pero se encontraron aspectos divergentes a las concepciones analizadas en la presente investigación, considerando las condiciones y diferencias individuales de los colaboradores, así como del mercado laboral, que podría derivar positivamente en un mayor involucramiento con la organización, caso contrario, la evasión de tareas o el abandono definitivo del cargo (Mobley, 1977).

En relación a la subcategoría ENG se distinguieron elementos destacados, caracterizados por una mayor implicación por parte de los trabajadores en relación a su desempeño laboral, a partir de la búsqueda de un mayor rendimiento en la 
realización de sus labores, favoreciendo una adhesión amplia con la organización y permitiendo que los colaboradores no presenten el síndrome de burnout. E ENG se caracteriza por ese estudio positivo en cuanto al trabajo; algunos puntos de concordancia encontrados en la definición de Schaufeli, Salanova, Gonzales y Bakker (2002) resaltan la energía, implicación, entusiasmo, orgullo y la fidelidad, llevando a los trabajadores a presentar mayor rendimiento laboral.

Una de las teorías pioneras de las relaciones humanas, con Elton Mayo como su principal exponente, fundamentó un enfoque centrado en el recurso humano y su potencial para las empresas como un elemento valioso y preponderante (Cruz, 2018), reafirmando cómo la CVL abordada desde la DHT es una estrategia de gestión que considera el talento humano como el aspecto más importante dentro de una organización; por ello, se interesa por propender la humanización y mejorar la dignidad de los colaboradores.

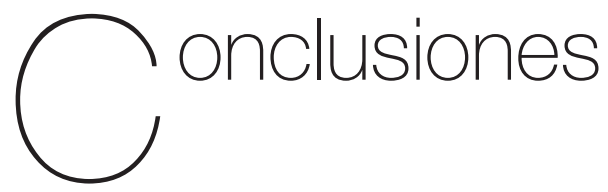

No se encontró un consenso frente al concepto de CVL que no involucre otro tipo de variables organizacionales tales como bienestar laboral, satisfacción laboral, engagement, dignificación y humanización del trabajo, puesto que es un constructo multidimensional y su concepción varía de acuerdo a la subjetividad de los trabajadores.
La CVL se ha convertido en un pilar fundamental para toda organización, de ahí su importancia por ampliar el estudio de este fenómeno y aportar a una comprensión más clara, holística y operacional, a través de un proceso de evaluación que reconozca un pluralismo metodológico y focos de análisis cualitativos y cuantitativos a través de la implementación de instrumentos y técnicas, para valorar todas aquellas facetas que componen la vida en el trabajo, tanto objetivas como subjetivas.

La naturaleza de los cargos y las funciones desarrolladas en el sector educación y salud tienen implicaciones en la CVL, en la salud y bienestar del trabajador e inciden en la productividad y competitividad de la organización.

Es importante el estudio de la SL desde el análisis de las distintas variables individuales y colectivas, tanto intrínsecas como extrínsecas del trabajador, relacionadas con aspectos específicos y situacionales de su ambiente de trabajo, así como el ENG, siendo un concepto relativamente nuevo dentro de las diversas variables organizacionales. Su identificación y abordaje debe ser tenido en cuenta dentro de los procesos de diagnóstico y propuestas de intervención de la CVL.

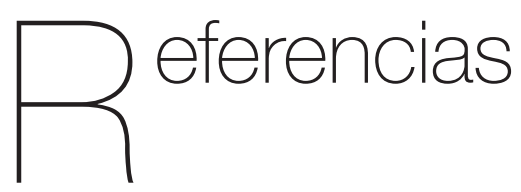

Armijo, M.l., \& Portalanza, C.A. (2017). Diagnóstico de la Calidad de vida laboral, Engagement y Burnout en trabajadores de 
una Institución de Educación Superior del Ecuador. Research Journal, 2(12), 153-165. Recuperado de https://dialnet.unirioja.es/ servlet/articulo?codigo=6259165

Bakker, A. B., \& Demerouti, E. (2013). La teoría de las demandas y los recursos laborales. Revista de Psicología del Trabajo y de las Organizaciones, 29(3), 107-115. https://doi. org/10.5093/tr2013a16

Blanch, J. (2011). Riesgos psicosociales desde la perspectiva de calidad de vida laboral. Acta Colombiana de Psicología, 14(2), 27-34. Recuperado de http://www. scielo.org.co/pdf/acp/v14n2/v14n2a03. pdf

Blanch, J. (2014). Calidad de vida laboral en hospitales y universidades mercantilizados. Papeles del Psicólogo, 35(1), 40-47. https://www.redalyc.org/ articulo.oa?id=778/77830184006

Caballero, M. F., \& Nieto, L. E. (2015). Nueva gestión pública en Colombia y bienestar laboral del profesorado universitario. Entramado, 11(1), 124-134. Recuperado de http://www.scielo.org.co/pdf/entra/ v11n1/v11n1a09.pdf

Cárcamo, H., Méndez, P., \& Rebolledo, A. (2009). Tendencias de los enfoques cualitativos y cuantitativos en artículos publicados en scientific library on line (Scielo). Paradígma, 30(2), 179200. Recuperado de http://ve.scielo. org/scielo.php?script=sci_arttext\&pid $=$ S1011-22512009000200012

Carlosama, D., Villota, N., Benavides, V., Villalobos, F., Hernández, E., \& Matabanchoy, S. (2019). Humanización de los servicios de salud en Iberoamérica: una revisión sistemática de la literatura.
Persona y Bioética 2019, 23(2), 245-262.

Recuperado de https://doi.org/10.5294/ pebi.2019.23.2.6

Casas, J., Repullo, J., Lorenzo, S., \& Cañas, J. (2002). Dimensiones y medición delacalidaddevidalaboralen profesionales sanitarios. Revista de Administración Sanitaria, 4(23), 527-544. Recuperado de https://www.researchgate.net/ publication/237316492_Dimensiones_y_ medicion_de_la_calidad_de_vida_ laboral_en_profesionales_sanitarios

Castro, E. \& Serna, M. (2016). Calidad del empleo en organizaciones de servicios de contact-center en Manizales Colombia. Revista Latinoamericana de Ciencias Sociales, 14(1), 205-219. Recuperado de http://www.scielo.org.co/pdf/rlcs/v14n1/ v14n1a14.pdf

Castro, P., Faria, V., Ferreira, M., \& De Fátima, A. (2012) Evaluación del bienestar en el trabajo entre los profesionales de enfermería de un hospital universitario. Revista Latino-Americana Emfermagem, 20(4), 1-9. Recuperado de http://www. scielo.br/pdf/rlae/v20n4/es_10.pdf

Cervantes, G., Blanch, J.M., \& Hermoso, D. (2010). Calidad de vida laboral en centros asistenciales de salud catalanes. Archivos de Prevención de Riesgos Laborales, 14(1), 13-19. Recuperado de http:// www.archivosdeprevencion.com/view_ document.php?tpd=2\&i=1966

Cetina, T., Escamilla, M., Centeno, G., \& Aguilar, C. (2018). Calidad de vida en el trabajo en operadores de autotransporte. Psicología desde el Caribe, 35, 80-97. Recuperado de https://dialnet.unirioja.es/ servlet/articulo?codigo=6816309 
Chaves, L. D., Grijalba, M.A., Bárcenas, D.R., Matabanchoy, S.M., \& Zambrano, C.A. (2017). Actitudes hacia la Calidad de vida laboral en trabajadores control de vía trasporte urbano. Tendencias, 18(2), 6985. Recuperado de https://dialnet.unirioja. es/servlet/articulo?codigo=6231285

Cruz, J. (2018). La calidad de vida laboral y el estudio del recurso humano: una reflexión sobre su relación con las variables organizacionales. Pensamiento \& Gestión, (45), 58-81. https://dx.doi.org/10.14482/ pege.45.10617

Dávila, H., Lombana, L., Matabanchoy, S., \& Zambrano, C. (2018). Factores psicosociales laborales inmersos en el contexto de los docentes en una Institución Educativa Estatal de San Juan de Pasto. Tendencias, 19(2), 138-160. https://doi.org/10.22267/rtend.181902.101

Echevarría, K., \& Santiago, R. (2017). Percepción del bienestar laboral de los empleados: estudio comparativo según género. Revista Internacional Administración y Finanzas, 10(2), 17-27. Recuperado de https://papers.ssrn.com/ sol3/papers.cfm?abstract_id=3039711

Fernández, A., Cuairan, M. \& Curbelo, R. (2016). Calidad de vida profesional de enfermería en urgencias de un hospital de referencia. Enfermería Global, 15(42), 376-385. Recuperado de http://scielo. isciii.es/scielo.php?script=sci_abstract\&pi $d=S 1695-61412016000200013$

Ferreira, I., Urrútia, G., \& Coello, P. A. (2011). Revisiones sistemáticas y meta análisis: bases conceptuales e interpretación. Revista Española de Cardiología, 64(4), 688-696. Recuperado de https://www. sciencedirect.com/science/article/abs/pii/ S0300893211004507?via\%3Dihub

Figueroa, H.A., Bastidas, C.F., Gonzales, W.R., Zambrano, C.A., \& Matabanchoy, S.M. (2017). Propiedades psicométricas de la Escala de Evaluación de Calidad de Vida Laboral en el sector trasporte urbano. Universidad y Salud, 19(3), 330-339. http:// dx.doi.org/10.22267/rus.171903.95

Flores, N., Jenaro, C., González, F., \& García, P.M. (2010). Análisis de la Calidad de vida laboral en trabajadores con discapacidad. EKAINA, 95-107. Recuperado de http:// www.zerbitzuan.net/documentos/ zerbitzuan/Analisis\%20calidad\%20 de\%20vida\%20laboral.pdf

García, M., \& Forero, C. (2016). Calidad de vida laboral y la disposición al cambio organizacional en funcionarios de empresas de la ciudad de BogotáColombia. Acta Colombiana de Psicología, 19(1), 79-90. Recuperado de http://www.scielo.org.co/pdf/acp/v19n1/ es_v19n1a05.pdf

García, M., Gonzales, R., Aldrete, M., Acosta, M. \& León, S. (2014). Relación entre calidad de vida en el trabajo y síntomas de estrés en el personal administrativo universitario. Ciencia y Trabajo, 16(50), 97-102. Recuperado de https://scielo. conicyt.cl/scielo.php?script=sci_arttext\&p id=S0718-24492014000200007

Garrido, J., Uribe, A. \& Blanch, J. (2011). Riesgos psicosociales desde la perspectiva de la calidad de vida laboral. Acta Colombiana de Psicología, 14(2), 2734. Recuperado de http://www.scielo.org. co/pdf/acp/v14n2/v14n2a03.pdf 
Gómez, C. \& Ponce, E. (2010). Modelo integrativo de la calidad de vida laboral percibida a partir de la inseguridad laboral, la jornada laboral y el salario, para hombres y mujeres trabajadores de empresas de la ciudad de Bogotá en diferentes rangos de edad. Psicología, Avances de la Disciplina, 4(2), 113-129. Recuperado de http://www.scielo.org.co/ scielo.php?pid=S1900238620100002000 10\&script=sci_abstract\&tlng=es

Gómez, M. (2010). Calidad de vida laboral en empleados temporales del Valle de Aburra Colombia. Revista Ciencias Estratégicas 18(24), 225-236. Recuperado de https://revistas.upb.edu.co/index.php/ cienciasestrategicas/article/view/708/624

Gómez, M., Galvis, L. \& Royuela, A. (2015). Calidad de vida laboral en Colombia: un índice multidimensional y difuso. Centro de Estudios Económicos Regionales (CEER), (230), 1-44. Recuperado de http://www. banrep.gov.co/docum/Lectura_finanzas/ pdf/dtser_230.pdf

González, R., Hidalgo, G., León, G., Contreras, M., Aldrete, M., Hidalgo, B. et al. (2015). Calidad de vida laboral en profesionales de la salud. Revista Calidad Asistencial, 18(33), 52-65. Recuperado de https://www.elsevier.es/es-revistarevista-calidad-asistencial-256-articulocalidad-vida-laboral-profesionales-saludS1134282X10001168

Granados, I (2011). Calidad de Vida Laboral: Historia, dimensiones y beneficios. Revista IIPSI, 14(2), 271-276. Recuperado de http://pepsic.bvsalud.org/pdf/ripsi/ v14n2/a14.pdf

Hernández, I., Lumbreras, M., Méndez, P., Rojas, E., Cervantes, M. \& Juárez,
C. (2017). Validación de una escala para medir la calidad de vida laboral en hospitales públicos de Tlaxcala. Salud Publica de México, 59(2), 183-192. http:// saludpublica.mx/index.php/spm/article/ view/7758

Hutton B., Catalá, F., \& Moher, D. (2016). La extensión de la declaración PRISMA para revisiones sistemáticas que incorporan metaanálisis en red: PRISMA-NMA. Medicina Clínica, 147(6), 262-266. http:// dx.doi.org/10.1016/j.medcli.2016.02.025

Macías, A.J., Gutiérrez, C., Carmona, F.J., \& Crespillo, D. (2016). Relación de la inteligencia emocional y la calidad de vida profesional con la consecución de objetivos laborales en el distrito de atención primaria Costa del Sol. Atención Primaria, 48(5), 301-307. https://doi. org/10.1016/j.aprim.2015.06.007

Marcantonio, C. (2017). Concepto y alcance del término Engagement. Requerimiento a los empleados. Notas de divulgación, 81-89. Recuperado de http://www.want. uji.es/wp-content/uploads/2017/11/2009_ Salanova-Schaufeli.pdf

Martínez, L., Oviedo, O. \& Luna, C. (2013). Condiciones de trabajo que impactan en la calidad de vida laboral. Salud Uninorte, 29(3), 542-560. Recuperado de http:// www.scielo.org.co/pdf/sun/v29n3/ v29n3a06.pdf

Martínez, M. \& Ros, M. (2010). De la calidad de vida laboral a los riesgos psicosociales evaluación de la calidad de vida laboral. Acciones e Investigaciones Sociales, 28, 5-55. Recuperado de https://dialnet.unirioja.es/servlet/ articulo?codigo $=3610086$ 
Miño, A. (2016). Calidad de vida laboral en docentes chilenos. Suma Psicológica, 13(2), 45-55. Recuperado de https://dialnet.unirioja.es/servlet/ articulo?codigo $=5763549$

Mobley, W. (1977). Vínculos intermedios en la relación entresatisfacción laboraly rotación de empleados. Revista de Psicología Aplicada, 62, 237-240. Recuperado de https://www.academia.edu/16173002/ The_validity_of_Mobleys_1977_model_of_ employee_turnover

Mobley, W., Griffeth, R., Hand, H. \& Meglino, B. (1979). Revisión y análisis conceptual del proceso de rotación de empleados. Boletín Psicológico, 86, 493-522. Recuperado de https://www.researchgate.net/ publication/200130259_A_Review_and_ Conceptual_Analysis_of_the_Employee_ Turnover_Process

Molina, J.O., Pérez, A. Y., Lizárraga, G., \& Larrañaga, A.M. (2018). Análisis de calidad de vida laboral y competitividad en empresas de servicios turísticos. 3C Empresa: Investigación y Pensamiento Crítico, 7(2), 44-67. http://dx.doi. org/10.17 993/3cemp.2018.070234.44-67/

Montoya, C., Méndez, J. \& Boyero, M. (2017). Trabajo digno y decente: una mirada desde la OIT para la generación de indicadores para las Pyme mexicanas y colombianas. Visión de Futuro, 21(2), 48106. Recuperado de http://revistacientifica. fce.unam.edu.ar/index.php?option=com_ content\&view $=$ article\&id $=444: 2018-04$ 04-21-34-30\&catid =185:2017-10-24-2244-21\&ltemid=95

Nadler, D., \& Lawler, E. (1983). Factores que influyen en el éxito de la calidad de la gestión laboral de los proyectos de vida laboral.
Diario de comportamiento Ocupacional, 1, 53-67. Recuperado de https://www. sciencedirect.com/science/article/abs/ pii/0090261683900037?via\%3Dihub

Organización Internacional del Trabajo [OIT]. (2019). Seguridad y salud en el centro del futuro del trabajo. Ginebra, Suiza: Oficina internacional del trabajo.

Palomo, G., Carrasco, J., Bastias, A., Méndez, M, \& Jiménez, A. (2015). Factores de riesgo psicosocial y satisfacción laboral en trabajadoras estacionales de Chile. Revista Panamericana de Salud Pública, 37(4/5), 301-307. Recuperado de https:// www.scielosp.org/pdf/rpsp/2015. v37n4-5/301-307/

Patlan, J. (2016). Construcción y propiedades psicométricas de la escala de calidad de vida en el trabajo. Ciencia y Trabajo, 18(56), 94-105. Recuperado de https:// scielo.conicyt.cl/scielo.php?script=sci_art text\&pid=S0718-24492016000200004

Pérez, D., Peralta, J. \& Fernández, P. (2014). Influencia de variables organizacionales en la calidad de vida laboral de funcionarios del sector público de salud en el extremo norte de Chile. Universitas Psychologica, 13(2), 551-561. Recuperado de https:// revistas.javeriana.edu.co/index.php/ revPsycho/article/view/4178

Pérez, D., \& Zurita, R. (2016). Calidad de vida laboral en trabajadores de salud pública en Chile. Salud y Sociedad, 5(2), 172-180. https://doi.org/10.22199/ S07187475.2014.0002.00002

Pérez, T., Campos, A., Negro, A. \& Caballero, F. (2011). Professional Burnout and Work Satisfaction in Spanish Allergists: Analysis of Working. Conditions in the Specialty. 
Journal of Investigational Allergology \& Clinical Immunology, 21(1), 13-21. Retrieved from https://www.researchgate. net/publication/50269483_Professional_ Burnout_and_Work_Satisfaction_in_ Spanish_Allergists_Analysis_of_Working_ Conditions_in_the_Specialty

Restrepo, F. E., \& López, A. M. (2013). Percepciones del entorno laboral de los profesores universitarios en un contexto de reorganización flexible en el trabajo. Cuadernos de Administración, 29(49), 55 63. Recuperado de http://www.scielo.org. co/pdf/cuadm/v29n49/v29n49a07.pdf

Rivera, D.A., Rivera, J.C., \& González, C. (2017). Validación de los cuestionarios CVP-35 y MBI-HSS para calidad de vida profesional y burnout en residentes. Investigación en Educación Médica, 6(21), 25-34. https:// doi.org/10.1016/j.riem.2016.05.010

Rodríguez, Y. (2016). Evaluación de la gestión de la Seguridad y Salud en el Trabajo. Un análisis de serie de casos organizacionales. Sotavento MBA, 28, 74-83. Recuperado de https://revistas. uexternado.edu.co/index.php/sotavento/ article/view/5483/6676

Romero, A. E., García, A., \& Brustad, R. J. (2009). Estado del arte y perspectiva actual del concepto de bienestar psicológico en psicología del deporte. Revista Latinoamericana de Psicología, 41(2), 335-347. https://www.redalyc.org/ pdf/805/80511496011.pdf

Salazar, J.G. (2018). La relación entre el apoyo organizacional percibido y la calidad de vida relacionada con el trabajo, con la implementación de un modelo de bienestar en la organización. SIGNOS,
10(2), 41-53. https://doi.org/10.15332/ s2145-1389.2018.0002.02

Salgado, J. \& Leria, F. (2018). Síndrome de burnout y calidad de vida profesional percibida según estilos de personalidad en profesores de educación primaria. Revista CES Psicología, 11, 69-89. Recuperado de http://revistas.ces.edu.co/index.php/ psicologia/article/view/4192

Schaufeli, M., Salanova, M., González, V., \& Bakker, A. (2002). The measurement of engagement and burnout: a two sample confirmatory factor analytic approach. Journal of Happiness Studies, 3, 71-92. Retrieved from https:// www.wilmarschaufeli.nl/publications/ Schaufeli/178.pdf

Segurado, A., \& Agulló, E. (2002). Calidad de vida laboral: hacia un enfoque integrador desde la Psicología Social. Psicothema, 14(4), 828-836. Recuperado de https://www.redalyc.org/articulo. oa?id=727/72714422

Suescún, S., Sarmiento, G., Álvarez, L. \& Lugo, M. (2016). Calidad de vida laboral en trabajadores de una Empresa Social del Estado de Tunja, Colombia. Revista Médica Risaralda, 22(1), 1417. Recuperado de https://revistas.utp. edu.co/index.php/revistamedica/article/ view/13631

Terán, A. \& Botero, C. (2011). El capitalismo organizacional: una mirada a la calidad de vida laboral en la docencia universitaria. Cuadernos de Administración, 27(46), 9-21. Recuperado de scielo.org.co/ scielo.php?script=sci_abstract\&pid =S0120-46452011000200002 
Torres, T.M., Munguía, J. A., \& Aranda, C. (2015). Concepciones culturales del concepto bienestar de personas con enfermedades crónicas y profesionales de la salud. Hacia la Promoción de la Salud, 20(1), 96-110. Recuperado de http://www. scielo.org.co/pdf/hpsal/v20n1/v20n1a07. pdf

Valero, I., \& Riaño, M. (2017). Contributions of occupational health and safety to the quality of working life: An analytical reflection. Ciencia y Tecnología para la
Salud Visual y Ocular, 15(2), 85-94. http:// dx.doi.org/10.19052/sv.4207

Vidal, J., Oliver, A., Galiana, L., \& Sansó, N. (2019). Calidad de vida laboral y autocuidado en enfermeras asistenciales con alta demanda emocional. Enfermería Clínica, 29(3), 186-194. https://doi. org/10.1016/j.enfcli.2018.06.004

Walton, R. (1973). Calidad de vida laboral: ¿qué es?. Revista Sloan Management, 15(1), 11-21. 